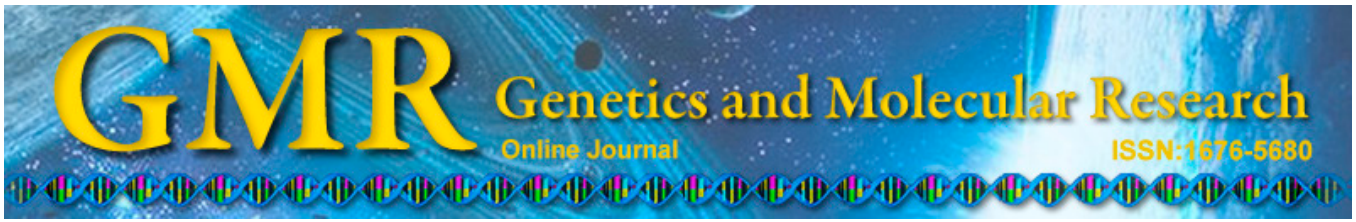

\title{
Compound heterozygosity of a novel exon 3 frameshift (p.R357P fs*24) mutation and Y486D mutation in exon 5 of the $U G T 1 A 1$ gene in a Thai infant with Crigler-Najjar syndrome type 2
}

\author{
L. Tesapirat, P. Nilyanimit, N. Wanlapakorn and Y. Poovorawan \\ Center of Excellence in Clinical Virology, Department of Pediatrics, \\ Faculty of Medicine, Chulalongkorn University, Bangkok, Thailand \\ Correspondence author: Y. Poovorawan \\ E-mail: Yong.P@chula.ac.th
}

Genet. Mol. Res. 14 (2): 3293-3299 (2015)

Received May 9, 2014

Accepted October 22, 2014

Published April 13, 2015

DOI http://dx.doi.org/10.4238/2015.April.13.8

\begin{abstract}
Mutations in the UGT1A1 gene cause CriglerNajjar syndrome $(\mathrm{CN})$, which causes non-hemolytic unconjugated hyperbilirubinemia, and is categorized as $\mathrm{CN} 1$ and $\mathrm{CN} 2$ according to the severity of bilirubin levels. The UGT1A1 gene is responsible for encoding the liver enzyme uridine diphosphate-glucuronosyltransferase, UGT1A1. This protein adds glucuronic acid to unconjugated bilirubin in bilirubin metabolism to form conjugated bilirubin. CN2 occurs when UGT1A1 activity is low, while CN1 is the absence of UGT1A1 activity; therefore, the CN2 phenotype is not as severe as that of CN1. Here, we report a novel allele of compound heterozygous mutations in UGT1A1 in a Thai male infant with clinical symptoms of CN2. The patient's compound heterozygosity was composed of a novel mutation, c.1069-1070insC, and the c.1456T $>\mathrm{G}$ mutation. The novel c.1069-1070insC mutation generated a premature stop codon in exon
\end{abstract}


4 (p.R357Pfs*24). The healthy parents were heterozygous for the c.1069-1070insC mutation (father) and c.1456T $>\mathrm{G}$ missense mutation (mother). Our results suggest that compound heterozygosity of the novel c.1069-1070insC and c.1456T $>\mathrm{G}(\mathrm{c} 211 \mathrm{G}>\mathrm{A})$ missense mutation in the UGT1A1 gene played a primary role in the development of CN2 unconjugated hyperbilirubinemia.

Key words: Crigler-Najjar syndrome; Hyperbilirubinemia; Infants; UGT1A1; Thai

\section{INTRODUCTION}

To date, approximately 130 mutations in the UGT1A1 gene have been reported as causes of Crigler-Najjar (CN) syndrome and Gilbert's syndrome (Canu et al., 2013). The TATA promoter plays a critical role in controlling the expression of the UGT1A1 gene. An insertion mutation with the nucleotide base "A" in the TATA promoter region leads to common mild hyperbilirubinemia, also known as Gilbert syndrome, with a total serum bilirubin level of 1-6 mg/dL (Bosma et al., 1995; Bayram et al., 2013). CN is related to inborn errors in metabolism and are inherited as autosomal recessive traits. These traits result from unconjugated hyperbilirubinemia because of defective bilirubin uridinediphosphate (UDP)glucuronosyltransferase (UGT1A1) activity (Iolascon et al., 2000). CN is classified as type 1 or 2 based on bilirubin levels, presence of kernicterus, phenobarbital response, and UGT1A1 enzyme-inducing agents (Costa et al., 2006; Bayram et al., 2013). Moreover, the clinical pathology of $\mathrm{CN}$ type 1 is characterized by the most severe manifestation, such as kernicterus, and can result in death when total serum bilirubin is $20-45 \mathrm{mg} / \mathrm{dL}$. This condition typically results from a lack of UGT1A1 activity and is non-responsive to phenobarbital administration. In contrast, $\mathrm{CN}$ type 2 is less severe and rarely causes kernicterus; total bilirubin levels for $\mathrm{CN}$ type 2 are typically $6-20 \mathrm{mg} / \mathrm{dL}$. UGT1A1 activity in CN type 2 is markedly reduced, but not absent, which can be improved by phenobarbital administration to decrease serum bilirubin levels by more than $25 \%$ (Arias et al., 1969; Labrune et al., 2002).

The UGT1A1 gene is one of the genes in the UGT1 gene complex (Iolascon et al., 2000; Gong et al., 2001). The UGT1 gene complex is located on chromosome 2q37 and encodes different proteins using alternative promoters with different first exons, but all share exons 2-5 (Bosma et al., 1994; Gong et al., 2001). The UGT1A1 gene consists of 5 exons, including exon A1 and exons 2-5 of the UGT1 gene complex. The UGT1A1 enzyme, a hepatic enzyme produced from the UGT1A1 gene, is involved in converting unconjugated bilirubin to a nontoxic form through the addition of glucuronic acid (Miners and Mackenzie, 1991).

In this study, we focused on CN type 2, which was diagnosed based on clinical manifestation and DNA sequencing of the UGT1A1 gene from genomic DNA of the patient and parents. Three mutations in the patient's UGT1A1 gene were identified, including c. $211 \mathrm{G}>\mathrm{A}$ (p.G71R) in exon 1, c.1069-1070insC (p.R357Pfs*24) in exon 3, and c.1456T $>$ G (p.G71R) in exon 5. Mutations in exons 3 and 5 were compound heterozygotes. The c.211G $>$ A (p.G71R) mutation is a common variation in the Japanese population (Akaba et al., 1999; Maruo et al., 2000); c.1456T $>$ G (p.Y486D) has also been identified (Nakagawa et al., 2011).

The purpose of this report was to examine the clinical presentation as well as to investigate and identify novel genetic mutations in UGT1A1 in a Thai infant with CN type 2. 


\section{MATERIAL AND METHODS}

This study was approved by the Institutional Review Board, Faculty of Medicine, Chulalongkorn University, Thailand. Written informed consent was obtained from the patient's parents.

\section{Patient}

A 1-year-old Thai male infant presented with jaundice at the age of 5 days. He was born at term, delivered by cesarean section because of cephalopelvic disproportion, and had a birth weight of $3636 \mathrm{~g}$. He was the second child of the family without a history of consanguineous marriage. The first child was healthy. At birth, he had 2 large cephalhematomas at both parietal areas, which caused an increase in total serum bilirubin of $18.9 \mathrm{mg} / \mathrm{dL}$ detected on the 5 th day of life. His thyroid function test was normal and a peripheral blood smear showed no evidence of hemolysis. The mother's and patient's blood groups were both $\mathrm{O}$, Rhesus factor-positive. Reticulocyte count and glucose-6-phosphate dehydrogenase level were normal. A direct Coomb's test and indirect Coomb's test were performed and the results were negative. He was treated with single phototherapy for 4 days and total serum bilirubin was successfully reduced to 8.2 $\mathrm{mg} / \mathrm{dL}$. He was discharged, but returned to the hospital 3 days later because of visible jaundice. Clinical examination showed resolving cephalhematomas. An initial investigation revealed an increase in total serum bilirubin to $19.8 \mathrm{mg} / \mathrm{dL}$ with predominantly unconjugated bilirubin of $19.7 \mathrm{mg} / \mathrm{dL}$. He was treated with single phototherapy for 5 days, and then referred to King Chulalongkorn Memorial Hospital for genetic testing to reach a final diagnosis.

Upon arrival at King Chulalongkorn Memorial Hospital, the patient had visible jaundice with normal growth and development. Physical examination was unremarkable. His total serum bilirubin level remained high $(15.8 \mathrm{mg} / \mathrm{dL})$. No significant increase in liver enzymes was recorded in this family as shown in Table 1.

\begin{tabular}{|c|c|c|c|c|}
\hline \multirow[t]{3}{*}{ Liver function test } & \multicolumn{2}{|c|}{ Patient } & \multirow[t]{3}{*}{ Father } & \multirow[t]{3}{*}{ Mother } \\
\hline & \multicolumn{2}{|c|}{ Phenobarbital treatment } & & \\
\hline & Before & After & & \\
\hline Albumin $(g / d L)$ & 4.0 & 4.7 & 4.7 & 4.1 \\
\hline Total bilirubin (g/dL) & 15.8 & 8.0 & 2.13 & 0.83 \\
\hline Direct bilirubin $(\mathrm{g} / \mathrm{dL})$ & 0.40 & 0.31 & 0.72 & 0.27 \\
\hline $\mathrm{AST}(\mathrm{U} / \mathrm{L})$ & 32 & 33 & 42 & 13 \\
\hline $\operatorname{ALT}(\mathrm{U} / \mathrm{L})$ & 18 & 17 & 100 & 11 \\
\hline $\operatorname{ALP}(\mathrm{U} / \mathrm{L})$ & 232 & 260 & 65 & 55 \\
\hline GGT(U/L) & 12 & 20 & 37 & 15 \\
\hline
\end{tabular}

AST $=$ aspartate aminotransferase $;$ ALT $=$ alanine aminotransferase $;$ ALP $=$ alkaline phosphatase GGT $=$ gamma glutamyltranspeptidase.

After arriving at King Chulalongkorn Memorial Hospital, the patient was treated with phenobarbital at a dosage of $3 \mathrm{mg} \cdot \mathrm{kg}^{-1} \cdot \mathrm{day}^{-1}$ for 4 weeks; total serum bilirubin level decreased significantly to $8.0 \mathrm{mg} / \mathrm{dL}$ after treatment (decrease of $49.4 \%$ ). Based on the presentation of unconjugated hyperbilirubinemia and decreased bilirubin levels after pheno- 
barbital treatment in the normally developed child, the patient was diagnosed with $\mathrm{CN}$ type 2. Blood samples of the patient and his family were collected and subjected to molecular genetic analysis.

\section{Methods}

One milliliter anti-coagulated (ethylenediamine tetraacetic acid-treated) blood sample was collected from the infant and his parents, respectively. Peripheral blood mononuclear cells were separated using lymphocyte separation medium (Wisent, Inc., Quebec, Canada). Genomic DNA was extracted from $100 \mu \mathrm{L}$ leukocytes using phenol-chloroform-isoamyl alcohol extraction and ethanol precipitation (Nilyanimit et al., 2013). The DNA pellet was stored at $-20^{\circ} \mathrm{C}$ until analysis.

For mutation screening, the promoter and 5 exons of UGT1A1 were amplified by polymerase chain reaction and direct sequencing, as described in a previous study (Nilyanimit et al., 2013). Nucleotide sequences obtained by direct sequencing were edited and assembled using SEQMAN (LASERGENE program package; DNASTAR, Inc., Madison, WI, USA) and subsequently subjected to Basic Local Alignment Search Tool analysis. The UGT1A1 accession No. NC_000002.11 from GenBank was used as the reference sequence, for which the "A" of the ATG translation initiation site represented nucleotide +1 .

\section{RESULTS}

Upon molecular genetic analysis of UGT1A1 in the patient and parents, we found that the patient had a normal wild-type A(TA)6TAA promoter, was homozygous for the $\mathrm{c} .211 \mathrm{G}>\mathrm{A}$ missense mutation, and showed the compound heterozygous mutation between c.10691070insC and c. 1456T $>$ G.

The first mutation, the c. $211 \mathrm{G}>\mathrm{A}$ missense mutation, was at position 211 in exon 1 and caused substitution of glycine with arginine at position 71 of the UGT1A1 protein (p.G71R). This mutation has been reported to be associated with Gilbert's syndrome in Japanese, Korean, and Chinese populations. In addition, its gene frequency is related to neonatal hyperbilirubinemia as a common variation among the Japanese populations. For instance, a higher frequency of mutated UGT1A1 gene is related to more severe hyperbilirubinemia as a clinical manifestation (Akaba et al., 1999).

The second mutation, the $\mathrm{c} .1456 \mathrm{~T}>\mathrm{G}$ missense mutation, was at position 1456 in exon 5 and led to substitution of tyrosine with aspartate at position 486 in the UGT1A1 enzyme (p.Y486D). Homozygosity of the second mutation has been reported as a cause of Gilbert's syndrome (Nakagawa et al., 2011). Moreover, the homozygous mutation has been associated with CN type 2. A homozygous mutation of c.T395C of UGT1D and c.G211A and c.T1456G of UGT1A1 has been reported to lead to CN type 2, but heterozygotes with these defective mutations were normal (Aono et al., 1993).

We also identified the novel mutation c.1069-1070insC in exon 3. Insertion of the base cytosine was observed between positions 1069 and 1070. This novel insertion mutation caused replacement of arginine with proline at position 357 of the UGT1A1 protein and caused a frameshift mutation for the next 24 amino acids, generating an early stop codon (p.R357Pfs*24) in exon 4. All mutations are shown in Figure 1. 


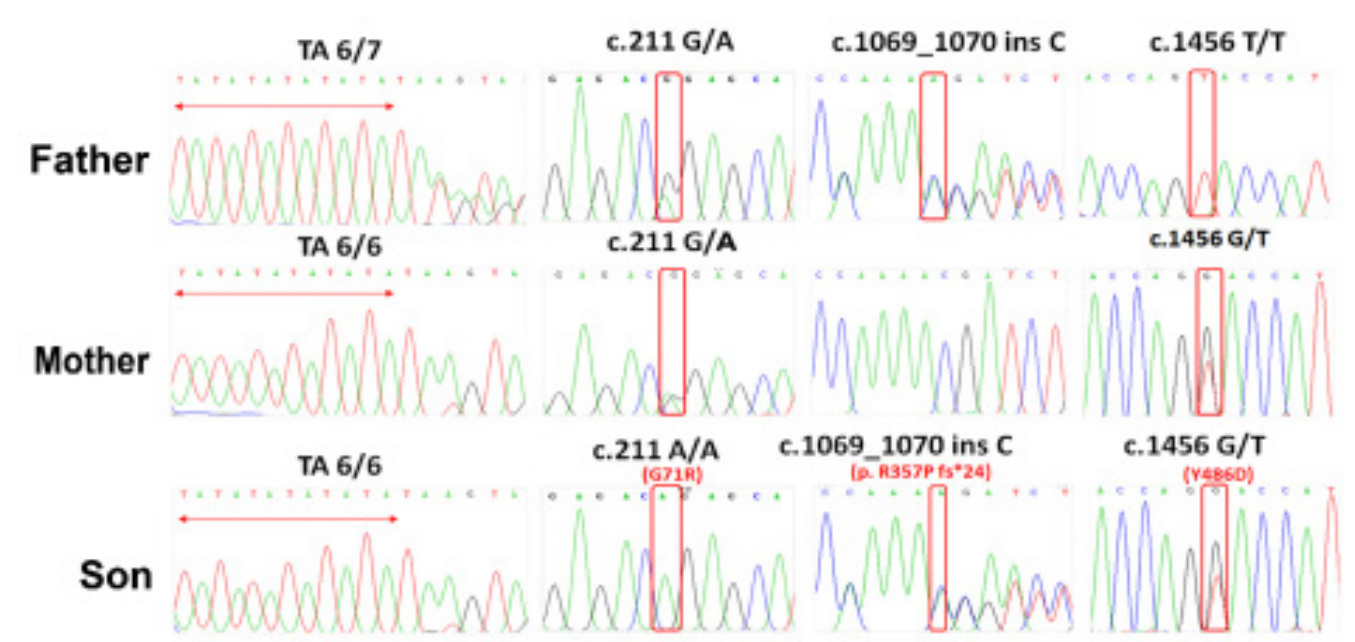

Figure 1. DNA sequence analysis of the patient and his parents. Nucleotide sequences showed heterozygous c. $211 \mathrm{G}>$ A mutations in the parents, a heterozygous c.1069-1070insC mutation in the father, a heterozygous c.1456 $\mathrm{T}>\mathrm{G}$ in the mother, and a homozygous c.211 G>A mutation and the compound heterozygous mutation of c.1456 $\mathrm{T}>\mathrm{G}$ and c.1069-1070insC in the patient.

Both parents were heterozygous for the c. $211 \mathrm{G}>\mathrm{A}$ missense mutation. The asymptomatic father was heterozygous for the c.1069-1070insC mutation and [A(TA)7TAA] promoter. The asymptomatic mother was heterozygous for c.211G $>$ A and c.1456T $>\mathrm{G}$, but had a normal wild-type promoter [A(TA)6TAA]. No mutations were detected in any other exon.

\section{DISCUSSION}

The patient's total serum bilirubin at 17 months of age was $15.8 \mathrm{~g} / \mathrm{dL}$. He was clinically well and was treated with $3 \mathrm{mg} \cdot \mathrm{kg}^{-1} \cdot \mathrm{day}^{-1}$ phenobarbital for 4 weeks. His total serum bilirubin decreased to $8.0 \mathrm{~g} / \mathrm{dL}$ (decrease of $49.37 \%$ ). Phenobarbital acts as an enzyme inducer for bilirubin conjugation, and treatment can reduce total serum bilirubin by at least $25 \%$ in CN type 2, but it cannot reduce total serum bilirubin to a normal baseline level (Arias et al., 1969; Labrune et al., 2002). Alkaline phosphatase was approximately $250 \mathrm{U} / \mathrm{L}$, which was normal for the patient's age (213-1148 U/L) because of bone development. He had no history of kernicterus. His stool was not pale and his development was normal. As a consequence of his clinical presentation, liver function test, and satisfactory response to phenobarbital, CN type 2 was diagnosed. Differential diagnoses included autoimmune hemolysis, G6PD, thalassemia, and Gilbert's syndrome.

Based on The Human Gene Mutation Database, more than 30 mutations are currently known to cause $\mathrm{CN}$ type 2, including missense, nonsense, a small deletion, and splicing mutations, of which missense and nonsense mutations comprise the majority (83.78\%). Missense mutations were found to be more common in $\mathrm{CN}$ type 2 than nonsense mutations, which always caused $\mathrm{CN}$ type 1 . Missense mutations were congruent with a less severe phenotype of CN type 2 as expected with residual UGT1A1 activity. Moreover, the spectrum of residual UGT1A1 activity accounted for the spectrum of phenotype severity in CN type 2. Based on 
molecular genetic analysis, the patient was not only homozygous for c.211G $>$ A (p.G71R) in exon 1 but was also compound heterozygous for c.1069-1070insC (p.R357P fs*24) in exon 3 on one allele and c.1456T $>\mathrm{G}$ (p.Y486D) in exon 5 on another allele.

The asymptomatic mother was heterozygous for c.211G $>$ A and c. $1456 \mathrm{~T}>\mathrm{G}$, but had a normal wild-type promoter [A(TA)6TAA] and normal liver function test. The asymptomatic father was heterozygous for [A(TA)7TAA] and c.1069-1070insC. His total bilirubin was mildly elevated because of slightly high direct bilirubin from an unknown cause.

Because of the less severe neurological pathology compared to $\mathrm{CN}$ type 1, specific treatment for hyperbilirubinemia may be unnecessary. CN type 1 is too severe to be treated with only phenobarbital; phototherapy (10-12 h/day) is necessary until liver transplantation can be performed (Kaufman et al., 1986; Sokal et al., 1995). In contrast, phenobarbital treatment alone is effective for $\mathrm{CN}$ type 2. Hence, liver transplantation was not required in this case. In addition, clofibrate administration, similarly to phenobarbital, is beneficial except for a few side effects (Gabilan et al., 1990). Pregnant women with CN type 2 can be treated with phenobarbital, but not with clofibrate (Passuello et al., 2009).

In conclusion, we report a case of $\mathrm{CN}$ type 2 in a 17-month-old Thai infant with normal growth development, but abnormal total bilirubin and visible jaundice. His condition improved after phenobarbital treatment. Molecular genetic analysis of the UGT1A1 gene confirmed homozygosity of the c. $211 \mathrm{G}>$ A mutation in exon 1 and compound heterozygous mutations of c.1456 $>\mathrm{G}$ in exon 5 and 1069-1070ins $\mathrm{C}$ in exon 3 . Thus, an early stop codon in one allele and a protein defect in another caused the UGT1A1 enzyme to be defective. In contrast, heterozygotes of these defective alleles were normal. Our results suggest that this compound heterozygous mutation played an important role in the development of CN type 2.

\section{Conflicts of interest}

The authors declare no conflict of interest.

\section{ACKNOWLEDGMENTS}

Research supported by the National Research University Project, Office of Higher Education Commission (\#HR1155A, \#WCU-001-HR-57, \#WCU-007-HR-57); the Center of Excellence in Clinical Virology, Chulalongkorn University; the Centenary Academic Development Project, Integrated Innovation Academic Center; the Chulalongkorn University Centenary Academic Development Project (\#CU56-HR01); the Ratchadaphiseksomphot Endowment Fund of Chulalongkorn University (\#RES560530093); the Outstanding Professor of the Thailand Research Fund (\#DPG5480002); the King Chulalongkorn Memorial Hospital Thai Red Cross Society; the Ratchadaphiseksomphot Endowment Fund, Chulalongkorn University; the MK Restaurant Company Limited; and The Siam Cement Pcl.

\section{REFERENCES}

Akaba K, Kimura T, Sasaki A, Tanabe S, et al. (1999). Neonatal hyperbilirubinemia and a common mutation of the bilirubin uridine diphosphate-glucuronosyltransferase gene in Japanese. J. Hum. Genet. 44: 22-25.

Aono S1, Yamada Y, Keino H, Hanada N, et al. (1993). Identification of defect in the genes for bilirubin UDP-glucuronosyltransferase in a patient with Crigler-Najjar syndrome type II. Biochem. Biophys. Res. Commun. 197: 1239-1244. 
Arias IM, Gartner LM, Cohen M, Ezzer JB, et al. (1969). Chronic nonhemolytic unconjugated hyperbilirubinemia with glucuronyl transferase deficiency. Clinical, biochemical, pharmacologic and genetic evidence for heterogeneity. Am. J. Med. 47: 395-409.

Bayram E, Öztürk Y, Hız S, Topçu Y, et al. (2013). Neurophysiological follow-up of two siblings with Crigler-Najjar syndrome type I and review of literature. Turk. J. Pediatr. 55: 349-353.

Bosma PJ, Seppen J, Goldhoorn B, Bakker C, et al. (1994). Bilirubin UDP-glucuronosyltransferase 1 is the only relevant bilirubin glucuronidating isoform in man. J. Biol. Chem. 269: 17960-17964.

Bosma PJ, Chowdhury JR, Bakker C, Gantla S, et al. (1995). The genetic basis of the reduced expression of bilirubin UDPglucuronosyltransferase 1 in Gilbert's syndrome. New Eng. J. Med. 333: 1171-1175.

Canu G, Minucci A, Zuppi C and Capoluongo E (2013). Gilbert and Crigler Najjar syndromes: an update of the UDPglucuronosyltransferase 1A1 (UGT1A1) gene mutation database. Blood Cells Mol. Dis. 50: 273-280.

Costa E, Vieira E, Martins M, Saraiva J, et al. (2006). Analysis of the UDP-glucuronosyltransferase gene in Portuguese patients with a clinical diagnosis of Gilbert and Crigler-Najjar syndromes. Blood Cells Mol. Dis. 36: 91-97.

Gabilan JC, Benattar C and Lindenbaum A (1990). Clofibrate treatment of neonatal jaundice. Pediatrics 86: 647-648.

Gong QH, Cho JW, Huang T, Potter C, et al. (2001). Thirteen UDP-glucuronosyltransferase genes are encoded at the human UGT1 gene complex locus. Pharmacogenetics 11: 357-368.

Iolascon A, Melon A, Coppola B, and Rosatelli MC (2000). Crigler-Najjar syndrome type II resulting from three different mutations in the bilirubin uridine 5'-diphosphate-glucuronosyltransferase (UGT1A1) gene. J. Med. Genet. 37: 712-713.

Kaufman SS, Wood RP, Shaw BW Jr, Markin RS, et al. (1986). Orthotopic liver transplantation for type I Crigler-Najjar syndrome. Hepatology 6: 1259-1262.

Labrune P, Myara A, Chalas J, Le Bihan B, et al. (2002). Association of a homozygous (TA)8 promoter polymorphism and a N400D mutation of UGT1A1 in a child with Crigler-Najjar type II syndrome. Hum. Mutat. 20: 399-401.

Maruo Y, Nishizawa K, Sato H, Sawa H, et al. (2000). Prolonged unconjugated hyperbilirubinemia associated with breast milk and mutations of the bilirubin uridine diphosphate-glucuronosyltransferase gene. Pediatrics 106: E59.

Miners JO and Mackenzie PI (1991). Drug glucuronidation in humans. Pharmacol. Ther. 51: 347-369.

Nakagawa T1, Mure T, Yusoff S, Ono E, et al. (2011). A homozygous mutation in UGT1A1 exon 5 may be responsible for persistent hyperbilirubinemia in a Japanese girl with Gilbert's syndrome. Kobe J. Med. 57: 26-31.

Nilyanimit P, Krasaelap A, Foonoi M, Chongsrisawat V, et al. (2013). Role of a homozygous A(TA) 7 TAA promoter polymorphism and an exon 1 heterozygous frameshift mutation UGT1A1 in Crigler-Najjar syndrome type II in a Thai neonate. Genet Mol Res.12:3391-3397.

Passuello V, Puhl AG, Wirth S, Steiner E, et al. (2009). Pregnancy outcome in maternal Crigler-Najjar syndrome type II: a case report and systematic review of the literature. Fetal Diagn. Ther. 26: 121-126.

Sokal EM, Silva ES, Hermans D, Reding R, et al. (1995). Orthotopic liver transplantation for Crigler-Najjar type I disease in six children. Transplantation 60: 1095-1098. 